\title{
A Turán-Kubilius type inequality on sum sets
}

by

\author{
JoËL Rivat (Marseille) and ANDrÁS SÁRKÖZY (Budapest)
}

1. Introduction. $\mathbb{N}, \mathbb{R}$ and $\mathbb{C}$ denote the set of positive integers, real numbers, resp. complex numbers. We write $\mathrm{e}(\alpha)=\exp (2 i \pi \alpha)$. The letter $p$ denotes a prime number. $\omega(n)$ denotes the number of distinct prime factors of $n$, while $\Omega(n)$ denotes the number of prime factors of $n$ counted with multiplicity.

In 1917 Hardy and Ramanujan [8] proved that for almost all positive integers $m \leq n$ the value of $\omega(m)$ is "near" $\log \log n$. Their proof was based on the estimate of the number of positive integers $m$ with $m \leq n$ and $\omega(m)=k$ for any fixed $k$. In 1934 Turán [13] proved in a simpler way that

$$
\sum_{m \leq n}(\omega(m)-\log \log n)^{2}=O(n \log \log n),
$$

from which the result of Hardy and Ramanujan follows immediately. Later Turán extended (1) to general additive arithmetic functions $f(n)$, and he showed that if $f(n)$ is a real-valued additive arithmetic function with

$$
f(p)=f\left(p^{2}\right)=\cdots=f\left(p^{k}\right)=\cdots
$$

for every prime number $p$ (in which case it is said to be strongly additive) and it is bounded:

$$
|f(p)|=O(1),
$$

then, writing

$$
A_{f}(n)=\sum_{p \leq n} \frac{f(p)}{p}
$$

we have

$$
\sum_{m \leq n}\left(f(m)-A_{f}(n)\right)^{2}=O\left(n A_{f}(n)\right) .
$$

2010 Mathematics Subject Classification: Primary 11K65; Secondary 11N64.

Key words and phrases: additive arithmetic function, probabilistic number theory, sum set, large sieve. 
In [9] Kubilius showed that Turán's conditions $f(n) \in \mathbb{R},(2)$ and (3) can be dropped, and still there is an inequality of type (5): if $f(n)$ is a complex-valued additive arithmetic function, $A_{f}(n)$ is defined by (4), and we also write

$$
D_{f}(n)=\left(\sum_{p^{\alpha} \leq n} \frac{\left|f\left(p^{\alpha}\right)\right|^{2}}{p^{\alpha}}\right)^{1 / 2}
$$

then

$$
\sum_{m \leq n}\left|f(m)-A_{f}(n)\right|^{2}=O\left(n D_{f}^{2}(n)\right) .
$$

This is called the Turán-Kubilius inequality.

In the last 25 years numerous papers have been written on the arithmetic properties of sum sets (see, e.g., [1 3], 4], [5], [6], [7, and [11, 12]). Typically, these results say that if $\mathcal{A}, \mathcal{B}$ are "large" subsets of $\{1, \ldots, n\}$ then a certain property of the sums simulates the behaviour of the consecutive integers $1, \ldots, n$. In particular, Elliott and Sárközy [5] showed that if $\mathcal{A}, \mathcal{B}$ are large subsets of $\{1, \ldots, n\}$, then the sums $a+b$ satisfy an Erdôs-Kac type theorem.

G. Halász (oral communication) asked whether the Turán-Kubilius inequality has a similar sum set analogue. We will show that, indeed, there is such an inequality which is, however, not quite as strong as (7): we will prove a similar result midway between Turán's and Kubilius's inequality.

2. The theorem and comments. We will prove the following theorem:

THEOREM 1. Let $f$ be a complex-valued additive arithmetic function, define

$$
K_{f}(m)=\max \left\{\left|f\left(p^{\alpha}\right)\right|: p \text { prime }, \alpha \in \mathbb{N}, p^{\alpha} \leq m\right\},
$$

let $A_{f}(n)$ be defined by (4), $C$ any fixed positive number, $n \in \mathbb{N}$ (with $n \rightarrow$ $+\infty)$ and $\mathcal{A}, \mathcal{B} \subseteq\{1, \ldots, n\}$ with

$$
\frac{n}{\sqrt{|\mathcal{A}||\mathcal{B}|}}<\exp (C \sqrt{\log \log n} \log \log \log n) .
$$

Then

$$
\frac{1}{|\mathcal{A}||\mathcal{B}|} \sum_{a \in \mathcal{A}} \sum_{b \in \mathcal{B}}\left|f(a+b)-A_{f}(2 n)\right|^{2}=O\left(C^{2} K_{f}^{2}(2 n) \log \log (2 n)\right) .
$$

Remarks. (i) If $f(n)=\omega(n)$ and $\mathcal{A}=\mathcal{B}=\mathbb{N}$, then the order of magnitude of the left hand side is the same as that of the function in the ordo term on the right hand side. This shows that in general $(10)$ is sharp apart from a constant factor. Indeed, one could construct much thinner sets $\mathcal{A}, \mathcal{B}$ (but not thinner than the lower bound implied by (9p) so that $10 p$ is sharp for these sets $\mathcal{A}, \mathcal{B}$ and $f(n)=\omega(n)$. 
(ii) Condition (9) is also sharp, i.e., to ensure that the left hand side of (10) is $O\left(K_{f}^{2}(2 n) \log \log (2 n)\right)$ one needs assumption (9). Indeed, fix a positive number $C$, let $Q$ denote the products of the primes not exceeding $(C / 2) \sqrt{\log \log n} \log \log \log n$, and let $\mathcal{A}=\mathcal{B}=\{i Q: i \leq n / Q\}$. Then it follows easily from the prime number theorem (or from a more elementary theorem) that (9) holds. Moreover, let $f(m)=\omega(m)$ (which implies $\left.K_{f}(2 n)=1\right)$. Then it can be shown that the left hand side of $(10)$ is

$$
\gg C^{\prime} K_{f}^{2}(2 n) \log \log (2 n)=C^{\prime} \log \log (2 n),
$$

with some $C^{\prime}=C^{\prime}(C)$ such that $C^{\prime} \rightarrow+\infty$ as $C \rightarrow+\infty$. (The reason is that for a typical pair $a=i Q \in \mathcal{A}, b=j Q \in \mathcal{B}$ we have $Q \mid a+b=$ $(i+j) Q$, thus every prime $p \leq(C / 2) \sqrt{\log \log n} \log \log \log n$ divides $a+b$. The number of these primes is $(1+o(1)) C \sqrt{\log \log n}$ so that their contribution makes a typical difference $f(a+b)-A_{f}(2 n)=\omega(a+b)-A_{\omega}(2 n)$ greater by $(1+o(1)) C \sqrt{\log \log n}$, which adds $C^{\prime} \log \log n$ to the left hand side of 10 . with some $C^{\prime}=C^{\prime}(C)$ such that $C^{\prime} \rightarrow+\infty$ as $C \rightarrow+\infty$.)

(iii) While Theorem 1 is sharp for $f(m)=\omega(m)$, it gives only a very weak upper bound for the left hand side of 10$)$ if $f(m)=\Omega(m)$. The reason is that the prime powers $p^{\alpha}$ with small $p$ and large $\alpha$ may influence the distribution of the values $\Omega(a+b)$ (with $a \in \mathcal{A}, b \in \mathcal{B}$ ) significantly. E.g. let $\alpha=\left\lfloor C^{\prime} \sqrt{\log \log n}\right\rfloor$, and set first $\mathcal{A}=\mathcal{B}=\left\{m: m \leq n, 2^{\alpha} \mid m\right\}$ and then $\mathcal{A}=\left\{m: m \leq n, 2^{\alpha} \mid m\right\}, \mathcal{B}=\left\{m: m \leq n, 2^{\alpha} \mid m+1\right\}$. In the first case the powers $2^{\beta}$ with $\beta \geq \alpha$ make a contribution $\gg \sqrt{\log \log n}$ to every value $f(a+b)=\Omega(a+b)(a \in \mathcal{A}, b \in \mathcal{B})$, while in the second case they make no contribution at all. In both cases the left hand side of $(9)$ is $<\exp \left(C^{\prime \prime} \sqrt{\log \log n}\right)$ so that a stronger inequality holds than (9); however, the left hand side of 10$)$ is $O(\log \log (2 n))$ in the first case, and in the second case it is $\neq O(\log \log (2 n))$ if $C^{\prime} \rightarrow+\infty$ slowly.

3. Structure of the proof. Let $\mathcal{P}$ denote the set of prime powers $p^{\alpha} \leq 2 n$, and write

$$
V=\frac{n}{\sqrt{|\mathcal{A}||\mathcal{B}|}}
$$

We split $\mathcal{P}$ into three parts: let

$$
\begin{aligned}
& \mathcal{P}_{1}=\left\{p^{\alpha}: p \leq V, p^{\alpha} \leq 2 n\right\} \\
& \mathcal{P}_{2}=\left\{p^{\alpha}: V<p, p^{\alpha} \leq \sqrt[4]{2 n}\right\} \\
& \mathcal{P}_{3}=\left\{p^{\alpha}: V<p, \sqrt[4]{2 n}<p^{\alpha} \leq 2 n\right\}
\end{aligned}
$$

so that $\mathcal{P}=\mathcal{P}_{1} \cup \mathcal{P}_{2} \cup \mathcal{P}_{3}$ and $\mathcal{P}_{i} \cap \mathcal{P}_{j}=\emptyset$ for $1 \leq i<j \leq 3$. Define the additive arithmetic functions $f_{1}(m), f_{2}(m), f_{3}(m)$ by 


$$
f_{i}\left(p^{\alpha}\right)=\left\{\begin{array}{ll}
f\left(p^{\alpha}\right) & \text { if } p^{\alpha} \in \mathcal{P}_{i} \\
0 & \text { if } p^{\alpha} \notin \mathcal{P}_{i}
\end{array} \quad(\text { for } i=1,2,3) .\right.
$$

Then clearly $f(m)=f_{1}(m)+f_{2}(m)+f_{3}(m)$. Thus by using the elementary inequality $\left|z_{1}+z_{2}+z_{3}\right|^{2} \leq 3\left(\left|z_{1}\right|^{2}+\left|z_{2}\right|^{2}+\left|z_{3}\right|^{2}\right.$ ) (where $z_{1}, z_{2}, z_{3}$ are any complex numbers) we can estimate the sum on the left hand side of 10 in the following way:

$$
\frac{1}{|\mathcal{A}||\mathcal{B}|} \sum_{a \in \mathcal{A}} \sum_{b \in \mathcal{B}}\left|f(a+b)-A_{f}(2 n)\right|^{2} \leq 3\left(T_{1}+T_{2}+T_{3}\right)
$$

where

$$
T_{i}=\frac{1}{|\mathcal{A}||\mathcal{B}|} \sum_{a \in \mathcal{A}} \sum_{b \in \mathcal{B}}\left|f_{i}(a+b)-\sum_{p \leq 2 n} \frac{f_{i}(p)}{p}\right|^{2} \quad(\text { for } i=1,2,3) .
$$

The crucial part of the proof is the estimate of $T_{2}$, which is based on the large sieve; this estimate will be carried out in Sections 4 and $5 . T_{1}$ will be estimated in Section 6, while the (nearly trivial) estimate of $T_{3}$ and the completion of the proof of Theorem 1 will be presented in Section 7 .

4. The estimate of $T_{2}$. Preliminary lemmas. For $\mathcal{A}, \mathcal{B} \subseteq\{1, \ldots, n\}$ and $m \in \mathbb{N}$ we define

$$
R(m)=\sum_{\substack{(a, b) \in \mathcal{A} \times \mathcal{B} \\ a+b \equiv 0 \bmod m}} 1-\frac{|\mathcal{A}||\mathcal{B}|}{m} .
$$

Lemma 1. For $n \in \mathbb{N}$ and $w \in\{1,2\}$ let

$$
\mathcal{M}_{n, w}=\left\{m: 1 \leq m \leq 2 n, 1 \leq \omega(m) \leq w, p^{\alpha} \| m \Rightarrow p^{\alpha} \in \mathcal{P}_{2}\right\} .
$$

Then for $n \geq 8$,

$$
\sum_{m \in \mathcal{M}_{n, w}}|R(m)| \leq 3 \kappa_{w}(2 n)|\mathcal{A}||\mathcal{B}|
$$

where

$$
\kappa_{w}(u)=\max _{\substack{k \leq u \\ 1 \leq \omega(k) \leq w}} \sum_{\substack{d \leq u / k \\ 1 \leq \omega(k d) \leq w}} \frac{1}{d}
$$

Moreover,

$$
\kappa_{1}(u) \leq 2, \quad \kappa_{2}(u) \leq 3+\sum_{p \leq u} \frac{1}{p}=O(\log \log u) .
$$

Proof. We first observe that we may assume

$$
V<\sqrt[4]{2 n}
$$

for otherwise $\mathcal{P}_{2}=\emptyset$, thus $\mathcal{M}_{n, w}=\emptyset$ and 15 is trivially true. We can write 


$$
\sum_{m \in \mathcal{M}_{n, w}}|R(m)|=\sum_{m \in \mathcal{M}_{n, w}} \frac{1}{m}\left|\sum_{1 \leq h<m}\left(\sum_{a \in \mathcal{A}} \mathrm{e}\left(\frac{h a}{m}\right)\right)\left(\sum_{b \in \mathcal{B}} \mathrm{e}\left(\frac{h b}{m}\right)\right)\right|
$$

and by Cauchy's inequality we get

$$
\begin{aligned}
\sum_{m \in \mathcal{M}_{n, w}}|R(m)| \leq & \left(\sum_{m \in \mathcal{M}_{n, w}} \frac{1}{m} \sum_{1 \leq h<m}\left|\sum_{a \in \mathcal{A}} \mathrm{e}\left(\frac{h a}{m}\right)\right|^{2}\right)^{1 / 2} \\
& \times\left(\sum_{m \in \mathcal{M}_{n, w}} \frac{1}{m} \sum_{1 \leq h<m}\left|\sum_{b \in \mathcal{B}} \mathrm{e}\left(\frac{h b}{m}\right)\right|^{2}\right)^{1 / 2} .
\end{aligned}
$$

Let us consider the first term on the right hand side of 19 . We arrange the summation according to the greatest common factor of $h$ and $m$ :

$$
\begin{aligned}
\sum_{m \in \mathcal{M}_{n, w}} \frac{1}{m} \sum_{1 \leq h<m}\left|\sum_{a \in \mathcal{A}} \mathrm{e}\left(\frac{h a}{m}\right)\right|^{2} & =\sum_{m \in \mathcal{M}_{n, w}} \frac{1}{m} \sum_{d \mid m} \sum_{\substack{1 \leq h<m \\
(h, m)=d}}\left|\sum_{a \in \mathcal{A}} \mathrm{e}\left(\frac{h a}{m}\right)\right|^{2} \\
& =\sum_{m \in \mathcal{M}_{n, w}} \frac{1}{m} \sum_{d \mid m} \sum_{\substack{1 \leq l<m / d \\
(l, m)=1}}\left|\sum_{a \in \mathcal{A}} \mathrm{e}\left(\frac{l a}{m / d}\right)\right|^{2} .
\end{aligned}
$$

Notice that this last sum is empty for $d=m$ so we may assume that $1 \leq d$ $<m$. We put $k=m / d$; observe that $k>1$. Since $k \mid m$ we have $1 \leq \omega(k) \leq w$ and $p^{\alpha} \| k \Rightarrow p^{\alpha} \in \mathcal{P}_{2}$, i.e. $k \in \mathcal{M}_{n, w}$. Hence changing the order of summation we get

$$
\sum_{m \in \mathcal{M}_{n, w}} \frac{1}{m} \sum_{1 \leq h<m}\left|\sum_{a \in \mathcal{A}} \mathrm{e}\left(\frac{h a}{m}\right)\right|^{2}=\sum_{k \in \mathcal{M}_{n, w}} \frac{1}{k} \sum_{\substack{d \leq 2 n / k \\ 1 \leq \omega(k d) \leq w}} \frac{1}{d} \sum_{\substack{1 \leq l<k \\(l, k)=1}}\left|\sum_{a \in \mathcal{A}} \mathrm{e}\left(\frac{l a}{k}\right)\right|^{2} .
$$

Using 16 we get

$$
\sum_{m \in \mathcal{M}_{n, w}} \frac{1}{m} \sum_{1 \leq h<m}\left|\sum_{a \in \mathcal{A}} \mathrm{e}\left(\frac{h a}{m}\right)\right|^{2} \leq \kappa_{w}(2 n) \sum_{k \in \mathcal{M}_{n, w}} \frac{1}{k} \sum_{\substack{1 \leq l<k \\(l, k)=1}}\left|\sum_{a \in \mathcal{A}} \mathrm{e}\left(\frac{l a}{k}\right)\right|^{2} .
$$

Let $z_{j}=2 n / 2^{j}$. For $k \in \mathcal{M}_{n, w}$ with $w \in\{1,2\}$, by the definition of $\mathcal{P}_{2}$ we have $V<k$ and $k \leq(\sqrt[4]{2 n})^{2}=\sqrt{2 n}$. It follows that if there is a $k \in \mathcal{M}_{n, w}$ with $z_{j+1}<k \leq z_{j}$, then $V<z_{j}=2 n / 2^{j}$ and $z_{j+1}=2 n / 2^{j+1}<\sqrt{2 n}$, whence $\sqrt{n / 2}<2^{j}<2 n / V$. Thus

$$
\begin{aligned}
\sum_{k \in \mathcal{M}_{n, w}} \frac{1}{k} \sum_{\substack{1 \leq l<k \\
(l, k)=1}}\left|\sum_{a \in \mathcal{A}} \mathrm{e}\left(\frac{l a}{k}\right)\right|^{2} & \leq \sum_{\sqrt{n / 2}<2^{j}<2 n / V} \frac{1}{z_{j}} \sum_{\substack { z_{j+1}<k \leq z_{j} \\
\begin{subarray}{c}{1 \leq l<k \\
(l, k)=1{ z _ { j + 1 } < k \leq z _ { j } \\
\begin{subarray} { c } { 1 \leq l < k \\
( l , k ) = 1 } }\end{subarray}}\left|\sum_{a \in \mathcal{A}} \mathrm{e}\left(\frac{l a}{k}\right)\right|^{2} .
\end{aligned}
$$


For fixed $j$ the points $l / k$ with $(l, k)=1$ are at least $\left(z_{j+1}\right)^{-2}$ spaced modulo 1 and $a \in \mathcal{A}$ satisfies $1 \leq a \leq n$, hence by the large sieve inequality (see for example [10]),

$$
\sum_{z_{j+1}<k \leq z_{j}} \sum_{\substack{1 \leq l<k \\(l, k)=1}}\left|\sum_{a \in \mathcal{A}} \mathrm{e}\left(\frac{l a}{k}\right)\right|^{2} \leq\left(n-1+z_{j+1}^{2}\right)|\mathcal{A}| .
$$

But

$$
\begin{aligned}
\sum_{\sqrt{n / 2}<2^{j}<2 n / V} \frac{1}{z_{j}}(n-1 & \left.+z_{j+1}^{2}\right) \\
& =\frac{n-1}{2 n} \sum_{\sqrt{n / 2}<2^{j}<2 n / V} 2^{j}+2 n \sum_{\sqrt{n / 2}<2^{j}<2 n / V} \frac{1}{2^{j+2}} \\
& \leq \frac{2 n}{V}+\sqrt{2 n},
\end{aligned}
$$

and by $(18)$,

$$
\frac{2 n}{V}+\sqrt{2 n}=\frac{2 n}{V}\left(1+\frac{V}{\sqrt{2 n}}\right) \leq \frac{2 n}{V}\left(1+\frac{\sqrt[4]{2 n}}{\sqrt{2 n}}\right) \leq \frac{3 n}{V}
$$

for $n \geq 8$, so that

$$
\sum_{m \in \mathcal{M}_{n, w}} \frac{1}{m} \sum_{1 \leq h<m}\left|\sum_{a \in \mathcal{A}} \mathrm{e}\left(\frac{h a}{m}\right)\right|^{2} \leq \frac{3 n}{V} \kappa_{w}(2 n)|\mathcal{A}| .
$$

We may replace $\mathcal{A}$ by $\mathcal{B}$ in the argument above and get

$$
\sum_{m \in \mathcal{M}_{n, w}} \frac{1}{m} \sum_{1 \leq h<m}\left|\sum_{b \in \mathcal{B}} \mathrm{e}\left(\frac{h b}{m}\right)\right|^{2} \leq \frac{3 n}{V} \kappa_{w}(2 n)|\mathcal{B}| .
$$

Finally, if we apply these two estimates in 19 and use the definition of $V$ given by (11), we get 15):

$$
\sum_{m \in \mathcal{M}_{n, w}}|R(m)| \leq \frac{3 n}{V} \kappa_{w}(2 n) \sqrt{|\mathcal{A}||\mathcal{B}|}=3 \kappa_{w}(2 n)|\mathcal{A}||\mathcal{B}| .
$$

It remains to prove (17). When $w=1$, we observe that $k$ in 16$)$ is a prime power $p^{\gamma}$, and $d$ must be a power of the same prime number $p$ (say $d=p^{\beta}$ ). More precisely, by the definition (16) we have

$$
\kappa_{1}(u)=\max _{\substack{k \leq u \\ \omega(k)=1}} \sum_{\substack{d \leq u / k \\ \omega(k d)=1}} \frac{1}{d}=\max _{\substack{p^{\gamma} \leq u \\ \gamma \geq 1}} \sum_{\substack{\beta \geq 0 \\ p^{\beta+\gamma} \leq u}} \frac{1}{p^{\beta}} \leq \max _{p} \sum_{\beta \geq 0} \frac{1}{p^{\beta}}=\max _{p} \frac{1}{1-1 / p}=2,
$$

which establishes the first statement of (17). The second is slightly more 
complicated. Observe that $\omega(k d) \geq \omega(d)$, hence by (16) we have

$$
\kappa_{2}(u)=\max \left(\max _{\substack{k \leq u \\ \omega(k)=1}} \sum_{\substack{d \leq u / k \\ 1 \leq \omega(k d) \leq 2}} \frac{1}{d}, \max _{\substack{k \leq u \\ \omega(k)=2}} \sum_{\substack{d \leq u / k \\ \omega(k d)=2}} \frac{1}{d}\right) .
$$

Now if $\omega(k)=2$ then $k=p^{\gamma} p^{\prime \gamma^{\prime}}$ and $d$ must be of the form $d=p^{\beta} p^{\prime \beta^{\prime}}$ with the same pair of prime numbers $\left(p, p^{\prime}\right)$ and $\beta, \beta^{\prime} \geq 0$. Therefore

$$
\max _{\substack{k \leq u \\ \omega(k)=2}} \sum_{\substack{d \leq u / k \\ \omega(k d)=2}} \frac{1}{d} \leq \max _{p \neq p^{\prime}} \sum_{\beta \geq 0} \frac{1}{p^{\beta}} \sum_{\beta^{\prime} \geq 0} \frac{1}{p^{\beta^{\prime}}}=\frac{1}{1-1 / 2} \frac{1}{1-1 / 3}=3 .
$$

Moreover

$$
\begin{aligned}
\max _{\substack{k \leq u \\
\omega(k)=1}} \sum_{\substack{d \leq u / k \\
1 \leq \omega(k d) \leq 2}} \frac{1}{d} \leq \max _{\substack{k \leq u \\
\omega(k)=1}} \sum_{\substack{d \leq u / k \\
\omega(k d)=1}} \frac{1}{d}+\max _{\begin{array}{c}
k \leq u \\
\omega(k)=1
\end{array}} \sum_{\substack{d \leq u / k \\
\omega(k d)=2}} \frac{1}{d} \\
=\kappa_{1}(u)+\max _{\substack{k \leq u \\
\omega(k)=1}} \sum_{\substack{d \leq u / k \\
\omega(k d)=2}} \frac{1}{d} .
\end{aligned}
$$

If $\omega(k)=1$ and $\omega(k d)=2$ this implies that $d$ is a prime power (say $d=p^{\beta}$ ) coprime to $k$. Hence if $\omega(k)=1$ we have

$$
\sum_{\substack{d \leq u / k \\ \omega(k d)=2}} \frac{1}{d} \leq \sum_{p \leq u / k} \sum_{\beta \geq 1} \frac{1}{p^{\beta}}=\sum_{p \leq u / k} \frac{1}{p}+\sum_{p \leq u / k} \frac{1}{p(p-1)} \leq \sum_{p \leq u / k} \frac{1}{p}+1 .
$$

Finally, we obtain

$$
\kappa_{2}(u) \leq \max \left(\kappa_{1}(u)+1+\sum_{p \leq u} \frac{1}{p}, 3\right) \leq 3+\sum_{p \leq u} \frac{1}{p},
$$

which is the second statement of (17).

Lemma 2. For any complex-valued additive arithmetic function $f_{2}$ such that $f_{2}\left(p^{\alpha}\right)=0$ whenever $p^{\alpha} \notin \mathcal{P}_{2}$ and $n \geq 8$ we have

$$
\left|\sum_{a \in \mathcal{A}} \sum_{b \in \mathcal{B}} f_{2}(a+b)-\right| \mathcal{A}|| \mathcal{B}\left|\sum_{p^{\alpha} \leq 2 n} \frac{f_{2}\left(p^{\alpha}\right)}{p^{\alpha}}\left(1-\frac{1}{p}\right)\right| \leq 14 K_{f_{2}}(2 n)|\mathcal{A}||\mathcal{B}|
$$

where $K_{f_{2}}$ is defined by (8).

Proof. Let

$$
S_{1}=\sum_{a \in \mathcal{A}} \sum_{b \in \mathcal{B}} f_{2}(a+b)
$$


Since $f_{2}$ is an additive arithmetic function we have

$$
S_{1}=\sum_{a \in \mathcal{A}} \sum_{b \in \mathcal{B}} \sum_{p^{\alpha} \| a+b} f_{2}\left(p^{\alpha}\right)=\sum_{p^{\alpha} \leq 2 n} f_{2}\left(p^{\alpha}\right) \sum_{\substack{(a, b) \in \mathcal{A} \times \mathcal{B} \\ p^{\alpha} \| a+b}} 1,
$$

and

$$
\sum_{\substack{(a, b) \in \mathcal{A} \times \mathcal{B} \\ p^{\alpha} \| a+b}} 1=\sum_{\substack{(a, b) \in \mathcal{A} \times \mathcal{B} \\ p^{\alpha} \mid a+b}} 1-\sum_{\substack{(a, b) \in \mathcal{A} \times \mathcal{B} \\ p^{\alpha+1} \mid a+b}} 1 .
$$

Using (14) we have

$$
\begin{aligned}
\sum_{\substack{(a, b) \in \mathcal{A} \times \mathcal{B} \\
p^{\alpha} \| a+b}} 1 & =\frac{|\mathcal{A}||\mathcal{B}|}{p^{\alpha}}-\frac{|\mathcal{A}||\mathcal{B}|}{p^{\alpha+1}}+R\left(p^{\alpha}\right)-R\left(p^{\alpha+1}\right) \\
& =\frac{|\mathcal{A}||\mathcal{B}|}{p^{\alpha}}\left(1-\frac{1}{p}\right)+R\left(p^{\alpha}\right)-R\left(p^{\alpha+1}\right)
\end{aligned}
$$

and

$S_{1}=|\mathcal{A}||\mathcal{B}| \sum_{p^{\alpha} \leq 2 n} \frac{f_{2}\left(p^{\alpha}\right)}{p^{\alpha}}\left(1-\frac{1}{p}\right)+\sum_{p^{\alpha} \leq 2 n} f_{2}\left(p^{\alpha}\right) R\left(p^{\alpha}\right)-\sum_{p^{\alpha} \leq 2 n} f_{2}\left(p^{\alpha}\right) R\left(p^{\alpha+1}\right)$,

so that using (8) and $f_{2}\left(p^{\alpha}\right)=0$ whenever $p^{\alpha} \notin \mathcal{P}_{2}$ we obtain

$$
\begin{aligned}
\left|S_{1}-\right| \mathcal{A}|| \mathcal{B} \mid \sum_{p^{\alpha} \leq 2 n} & \frac{f_{2}\left(p^{\alpha}\right)}{p^{\alpha}}\left(1-\frac{1}{p}\right) \mid \\
& \leq K_{f_{2}}(2 n)\left(\sum_{p^{\alpha} \in \mathcal{P}_{2}}\left|R\left(p^{\alpha}\right)\right|+\sum_{p^{\alpha} \in \mathcal{P}_{2}}\left|R\left(p^{\alpha+1}\right)\right|\right) .
\end{aligned}
$$

By (15) and (17) we have

$$
\sum_{p^{\alpha} \in \mathcal{P}_{2}}\left|R\left(p^{\alpha}\right)\right| \leq 6|\mathcal{A}||\mathcal{B}|
$$

and

$$
\begin{aligned}
\sum_{p^{\alpha} \in \mathcal{P}_{2}}\left|R\left(p^{\alpha+1}\right)\right| & =\sum_{\substack{p^{\alpha} \in \mathcal{P}_{2} \\
p^{\alpha+1} \notin \mathcal{P}_{2}}}\left|R\left(p^{\alpha+1}\right)\right|+\sum_{p^{\alpha+1} \in \mathcal{P}_{2}}\left|R\left(p^{\alpha+1}\right)\right| \\
& \leq \sum_{\substack{p^{\alpha} \in \mathcal{P}_{2} \\
p^{\alpha+1} \notin \mathcal{P}_{2}}}\left|R\left(p^{\alpha+1}\right)\right|+6|\mathcal{A}||\mathcal{B}| .
\end{aligned}
$$

Using (14) we have

$$
\left|R\left(p^{\alpha+1}\right)\right| \leq \sum_{\substack{(a, b) \in \mathcal{A} \times \mathcal{B} \\ a+b \equiv 0 \bmod p^{\alpha+1}}} 1+\frac{|\mathcal{A}||\mathcal{B}|}{p^{\alpha+1}}
$$


and counting trivially we can write

$$
\sum_{\substack{(a, b) \in \mathcal{A} \times \mathcal{B} \\ a+b \equiv 0 \bmod p^{\alpha+1}}} 1 \leq \min \left(\frac{n|\mathcal{A}|}{p^{\alpha+1}}, \frac{n|\mathcal{B}|}{p^{\alpha+1}}\right) \leq \frac{n}{p^{\alpha+1}} \sqrt{|\mathcal{A}||\mathcal{B}|},
$$

so that

$$
\sum_{\substack{p^{\alpha} \in \mathcal{P}_{2} \\ p^{\alpha+1} \notin \mathcal{P}_{2}}}\left|R\left(p^{\alpha+1}\right)\right| \leq(n \sqrt{|\mathcal{A}||\mathcal{B}|}+|\mathcal{A}||\mathcal{B}|) \sum_{\substack{p^{\alpha} \in \mathcal{P}_{2} \\ p^{\alpha+1} \notin \mathcal{P}_{2}}} \frac{1}{p^{\alpha+1}} .
$$

We will show that

$$
\sum_{\substack{p^{\alpha} \in \mathcal{P}_{2} \\ p^{\alpha+1} \notin \mathcal{P}_{2}}} \frac{1}{p^{\alpha+1}} \leq \frac{1}{V}
$$

Indeed, if $p^{\alpha} \in \mathcal{P}_{2}$ and $p^{\alpha+1} \notin \mathcal{P}_{2}$ then by the definition of $\mathcal{P}_{2}$ we have $V<p$ and $p^{\alpha} \leq \sqrt[4]{2 n}<p^{\alpha+1}$ so that $\alpha$ is uniquely defined. Hence

$$
\sum_{\substack{p^{\alpha} \in \mathcal{P}_{2} \\ p^{\alpha+1} \notin \mathcal{P}_{2}}} \frac{1}{p^{\alpha+1}} \leq \sum_{p>V} \frac{1}{p^{2}}
$$

But for $n_{0} \in \mathbb{N}$,

$$
\sum_{n \geq n_{0}} \frac{1}{(2 n+1)^{2}} \leq \sum_{n \geq n_{0}} \frac{1}{4}\left(\frac{1}{n}-\frac{1}{n+1}\right) \leq \frac{1}{4 n_{0}},
$$

so that if $V \geq 2$ then

$$
\sum_{p>V} \frac{1}{p^{2}}=\sum_{p \geq\lfloor V\rfloor+1} \frac{1}{p^{2}} \leq \sum_{n \geq\lfloor V\rfloor / 2} \frac{1}{(2 n+1)^{2}} \leq \frac{1}{2\lfloor V\rfloor} \leq \frac{1}{2 V-2} \leq \frac{1}{V},
$$

and if $1 \leq V<2$ then

$$
\sum_{p>V} \frac{1}{p^{2}}=\sum_{p \geq 2} \frac{1}{p^{2}} \leq \frac{1}{4}+\sum_{n \geq 1} \frac{1}{(2 n+1)^{2}} \leq \frac{1}{4}+\frac{1}{4} \leq \frac{1}{V} .
$$

By these estimates and the definition of $V$ given by (11) we get

$$
\sum_{\substack{p^{\alpha} \in \mathcal{P}_{2} \\ p^{\alpha+1} \notin \mathcal{P}_{2}}}\left|R\left(p^{\alpha+1}\right)\right| \leq \frac{n \sqrt{|\mathcal{A}||\mathcal{B}|}+|\mathcal{A}||\mathcal{B}|}{V} \leq 2|\mathcal{A}||\mathcal{B}|
$$

The sum of the last three upper bounds above gives

$$
\sum_{p^{\alpha} \in \mathcal{P}_{2}}\left|R\left(p^{\alpha}\right)\right|+\sum_{p^{\alpha} \in \mathcal{P}_{2}}\left|R\left(p^{\alpha+1}\right)\right| \leq 14|\mathcal{A}||\mathcal{B}| .
$$

Now (20) follows from (23) and (25). 
Lemma 3. For any complex-valued additive arithmetic function $f_{2}$ such that $f_{2}\left(p^{\alpha}\right)=0$ whenever $p^{\alpha} \notin \mathcal{P}_{2}$, and $n \geq 8$, we have

$$
\begin{aligned}
\left.\left|\sum_{a \in \mathcal{A}} \sum_{b \in \mathcal{B}}\right| f_{2}(a+b)\right|^{2}-|\mathcal{A}||\mathcal{B}| \mid \sum_{p^{\alpha} \leq 2 n} & \left.\frac{f_{2}\left(p^{\alpha}\right)}{p^{\alpha}}\left(1-\frac{1}{p}\right)\right|^{2} \mid \\
& \ll K_{f_{2}}^{2}(2 n)|\mathcal{A}||\mathcal{B}| \log \log (2 n)
\end{aligned}
$$

where $K_{f_{2}}$ is defined by (8).

Proof. Let

$$
S_{2}=\sum_{a \in \mathcal{A}} \sum_{b \in \mathcal{B}}\left|f_{2}(a+b)\right|^{2} .
$$

Since $f_{2}$ is an additive arithmetic function we can write

$$
S_{2}=\sum_{a \in \mathcal{A}} \sum_{b \in \mathcal{B}}\left|\sum_{p^{\alpha} \| a+b} f_{2}\left(p^{\alpha}\right)\right|^{2}
$$

and expanding the square we get

$$
S_{2}=\sum_{p^{\alpha} \leq 2 n}\left|f_{2}\left(p^{\alpha}\right)\right|^{2} \sum_{\substack{(a, b) \in \mathcal{A} \times \mathcal{B} \\ p^{\alpha} \| a+b}} 1+\sum_{\substack{p^{\alpha} q^{\beta} \leq 2 n \\ p \neq q}} f_{2}\left(p^{\alpha}\right) \overline{f_{2}\left(q^{\beta}\right)} \sum_{\substack{(a, b) \in \mathcal{A} \times \mathcal{B} \\ p^{\alpha}\left\|a+b \\ q^{\beta}\right\| a+b}} 1 .
$$

First we will give an upper bound for the first term. Using $\sqrt{22}$ we can write

$$
\begin{aligned}
\sum_{p^{\alpha} \leq 2 n}\left|f_{2}\left(p^{\alpha}\right)\right|^{2} & \sum_{\substack{(a, b) \in \mathcal{A} \times \mathcal{B} \\
p^{\alpha} \| a+b}} 1 \\
& =\sum_{p^{\alpha} \leq 2 n}\left|f_{2}\left(p^{\alpha}\right)\right|^{2}\left(\frac{|\mathcal{A}||\mathcal{B}|}{p^{\alpha}}\left(1-\frac{1}{p}\right)+R\left(p^{\alpha}\right)-R\left(p^{\alpha+1}\right)\right) \\
& \leq K_{f_{2}}^{2}(2 n) \sum_{p^{\alpha} \in \mathcal{P}_{2}}\left(\frac{|\mathcal{A}||\mathcal{B}|}{p^{\alpha}}\left(1-\frac{1}{p}\right)+\left|R\left(p^{\alpha}\right)\right|+\left|R\left(p^{\alpha+1}\right)\right|\right) .
\end{aligned}
$$

Now

$$
\begin{aligned}
\sum_{p^{\alpha} \in \mathcal{P}_{2}} \frac{|\mathcal{A}||\mathcal{B}|}{p^{\alpha}}\left(1-\frac{1}{p}\right) & \leq|\mathcal{A}||\mathcal{B}| \sum_{p \leq 2 n} \sum_{\alpha \geq 1} \frac{1}{p^{\alpha}}\left(1-\frac{1}{p}\right) \\
& =|\mathcal{A}||\mathcal{B}| \sum_{p \leq 2 n} \frac{1}{p}=O(|\mathcal{A}||\mathcal{B}| \log \log (2 n))
\end{aligned}
$$

while by (25) we have

$$
\sum_{p^{\alpha} \in \mathcal{P}_{2}}\left|R\left(p^{\alpha}\right)\right|+\sum_{p^{\alpha} \in \mathcal{P}_{2}}\left|R\left(p^{\alpha+1}\right)\right| \leq 14|\mathcal{A}||\mathcal{B}| .
$$


Using these two estimates we obtain, from (28),

$$
S_{2}=\sum_{\substack{p^{\alpha} q^{\beta} \leq 2 n \\ p \neq q}} f_{2}\left(p^{\alpha}\right) \overline{f_{2}\left(q^{\beta}\right)} \sum_{\substack{(a, b) \in \mathcal{A} \times \mathcal{B} \\ p^{\alpha}\left\|a+b \\ q^{\beta}\right\| a+b}} 1+O\left(K_{f_{2}}^{2}(2 n)|\mathcal{A}||\mathcal{B}| \log \log (2 n)\right)
$$

For $q \neq p$,

$$
\sum_{\substack{(a, b) \in \mathcal{A} \times \mathcal{B} \\ p^{\alpha}\left\|a+b \\ q^{\beta}\right\| a+b}} 1=\sum_{\substack{(a, b) \in \mathcal{A} \times \mathcal{B} \\ p^{\alpha} q^{\beta} \mid a+b}} 1-\sum_{\substack{(a, b) \in \mathcal{A} \times \mathcal{B} \\ p^{\alpha+1} q^{\beta} \mid a+b}} 1-\sum_{\substack{(a, b) \in \mathcal{A} \times \mathcal{B} \\ p^{\alpha} q^{\beta+1} \mid a+b}} 1+\sum_{\substack{(a, b) \in \mathcal{A} \times \mathcal{B} \\ p^{\alpha+1} q^{\beta+1} \mid a+b}} 1,
$$

thus using (14) we get

$$
\begin{aligned}
& \sum_{\substack{(a, b) \in \mathcal{A} \times \mathcal{B} \\
p^{\alpha}\left\|a+b \\
q^{\beta}\right\| a+b}} 1=\frac{|\mathcal{A}||\mathcal{B}|}{p^{\alpha} q^{\beta}}\left(1-\frac{1}{p}\right)\left(1-\frac{1}{q}\right) \\
& \quad+R\left(p^{\alpha} q^{\beta}\right)-R\left(p^{\alpha+1} q^{\beta}\right)-R\left(p^{\alpha} q^{\beta+1}\right)+R\left(p^{\alpha+1} q^{\beta+1}\right) .
\end{aligned}
$$

Writing

$$
S_{2}^{\prime}=\sum_{\substack{\alpha \\ p^{\alpha} q^{\beta} \leq 2 n \\ p \neq q}} \frac{f_{2}\left(p^{\alpha}\right) \overline{f_{2}\left(q^{\beta}\right)}}{p^{\alpha} q^{\beta}}\left(1-\frac{1}{p}\right)\left(1-\frac{1}{q}\right)
$$

and

$$
R_{2}=\sum_{\substack{p^{\alpha} \in \mathcal{P}_{2} \\ q^{\beta} \in \mathcal{P}_{2} \\ p \neq q}}\left(\left|R\left(p^{\alpha} q^{\beta}\right)\right|+\left|R\left(p^{\alpha+1} q^{\beta}\right)\right|+\left|R\left(p^{\alpha} q^{\beta+1}\right)\right|+\left|R\left(p^{\alpha+1} q^{\beta+1}\right)\right|\right),
$$

we deduce from 29$)$ and 30 that

$$
\left|S_{2}-\right| \mathcal{A}|| \mathcal{B}\left|S_{2}^{\prime}\right| \ll K_{f_{2}}^{2}(2 n)\left(|\mathcal{A}||\mathcal{B}| \log \log (2 n)+R_{2}\right) .
$$

By (15) and (17) we have

$$
\sum_{\substack{p^{\alpha} \in \mathcal{P}_{2}, q^{\beta} \in \mathcal{P}_{2} \\ p \neq q}}\left|R\left(p^{\alpha} q^{\beta}\right)\right| \ll|\mathcal{A}||\mathcal{B}| \log \log (2 n) .
$$

Using (14) we have

$$
\left|R\left(p^{\alpha+1} q^{\beta}\right)\right| \leq \sum_{\substack{(a, b) \in \mathcal{A} \times \mathcal{B} \\ a+b \equiv 0 \bmod p^{\alpha+1} q^{\beta}}} 1+\frac{|\mathcal{A}||\mathcal{B}|}{p^{\alpha+1} q^{\beta}}
$$


and counting trivially we can write

$$
\sum_{\substack{(a, b) \in \mathcal{A} \times \mathcal{B} \\ a+b \equiv 0 \bmod p^{\alpha+1} q^{\beta}}} 1 \leq \min \left(\frac{n|\mathcal{A}|}{p^{\alpha+1} q^{\beta}}, \frac{n|\mathcal{B}|}{p^{\alpha+1} q^{\beta}}\right) \leq \frac{n}{p^{\alpha+1} q^{\beta}} \sqrt{|\mathcal{A}||\mathcal{B}|},
$$

thus using (24)

$$
\begin{aligned}
\sum_{\substack{p^{\alpha} \in \mathcal{P}_{2} \\
p^{\alpha+1} \notin \mathcal{P}_{2}}} \sum_{\substack{q_{2} \in \mathcal{P}_{2} \\
q \neq p}}\left|R\left(p^{\alpha+1} q^{\beta}\right)\right| \\
\qquad n \sqrt{|\mathcal{A}||\mathcal{B}|} \sum_{\substack{p^{\alpha} \in \mathcal{P}_{2} \\
p^{\alpha+1} \notin \mathcal{P}_{2}}} \frac{1}{p^{\alpha+1}} \sum_{\substack{q^{\beta} \in \mathcal{P}_{2} \\
q \neq p}} \frac{1}{q^{\beta}} \ll \frac{n \sqrt{|\mathcal{A}||\mathcal{B}|}}{V} \log \log n,
\end{aligned}
$$

and by the definition of $V$ given by (11) we get

$$
\sum_{\substack{p^{\alpha} \in \mathcal{P}_{2} \\ p^{\alpha+1} \notin \mathcal{P}_{2}}} \sum_{\substack{q^{\beta} \in \mathcal{P}_{2} \\ q \neq p}}\left|R\left(p^{\alpha+1} q^{\beta}\right)\right| \ll|\mathcal{A}||\mathcal{B}| \log \log n .
$$

Similarly

$$
\sum_{\substack{q^{\beta} \in \mathcal{P}_{2} \\ q^{\beta+1} \notin \mathcal{P}_{2}}} \sum_{\substack{p^{\alpha} \in \mathcal{P}_{2} \\ q \neq p}}\left|R\left(p^{\alpha} q^{\beta+1}\right)\right| \ll|\mathcal{A}||\mathcal{B}| \log \log n
$$

and

$$
\sum_{\substack{p^{\alpha} \in \mathcal{P}_{2} \\ p^{\alpha+1} \notin \mathcal{P}_{2}}} \sum_{\substack{q^{\beta} \in \mathcal{P}_{2} \\ q^{\beta+1} \notin \mathcal{P}_{2}}}\left|R\left(p^{\alpha+1} q^{\beta+1}\right)\right| \ll|\mathcal{A}||\mathcal{B}| .
$$

Thus it follows from (31) that

$$
\left|S_{2}-\right| \mathcal{A}|| \mathcal{B}\left|S_{2}^{\prime}\right| \ll K_{f_{2}}^{2}(2 n)|\mathcal{A}||\mathcal{B}| \log \log (2 n) .
$$

In order to prove (26) it is sufficient to show that

$$
\left|\sum_{p^{\alpha} \leq 2 n} \frac{f_{2}\left(p^{\alpha}\right)}{p^{\alpha}}\left(1-\frac{1}{p}\right)\right|^{2}=S_{2}^{\prime}+O\left(K_{f_{2}}^{2}(2 n)\right) .
$$

We write

$$
\begin{aligned}
&\left|\sum_{p^{\alpha} \leq 2 n} \frac{f_{2}\left(p^{\alpha}\right)}{p^{\alpha}}\left(1-\frac{1}{p}\right)\right|^{2}=\sum_{\substack{p^{\alpha} \leq 2 n \\
q^{\beta} \leq 2 n}} \frac{f_{2}\left(p^{\alpha}\right) \overline{f_{2}\left(q^{\beta}\right)}}{p^{\alpha} q^{\beta}}\left(1-\frac{1}{p}\right)\left(1-\frac{1}{q}\right) \\
&=\sum_{\substack{p^{\alpha} \leq 2 n \\
q^{\beta} \leq 2 n \\
p=q}} \cdots+\sum_{\substack{p^{\alpha} q^{\beta} \leq 2 n \\
p \neq q}} \cdots+\sum_{\substack{p^{\alpha} \leq 2 n \\
q^{\beta} \leq 2 n \\
p^{\alpha} q^{\beta}>2 n \\
p \neq q}} \cdots . \\
& \substack{p \neq q \\
p=q}
\end{aligned}
$$


By the definition of $f_{2}$ we have $f_{2}\left(p^{\alpha}\right) \overline{f_{2}\left(q^{\beta}\right)} \neq 0$ only if $p^{\alpha} \leq \sqrt[4]{2 n}$ and $q^{\beta} \leq \sqrt[4]{2 n}$. This implies that the third sum above is empty $\left(p^{\alpha} q^{\beta}>2 n\right.$ is not possible). $S_{2}^{\prime}$ is the second sum above. The first sum can be majorized easily:

$$
\begin{aligned}
& \left|\sum_{\substack{p^{\alpha} \leq 2 n \\
q^{\beta} \leq 2 n \\
p=q}} \frac{f_{2}\left(p^{\alpha}\right) \overline{f_{2}\left(q^{\beta}\right)}}{p^{\alpha} q^{\beta}}\left(1-\frac{1}{p}\right)\left(1-\frac{1}{q}\right)\right| \\
& \quad \leq K_{f_{2}}^{2}(2 n) \sum_{p}\left(1-\frac{1}{p}\right)^{2} \sum_{\alpha \geq 1} \frac{1}{p^{\alpha}} \sum_{\beta \geq 1} \frac{1}{p^{\beta}}=K_{f_{2}}^{2}(2 n) \sum_{p} \frac{1}{p^{2}}=O\left(K_{f_{2}}^{2}(2 n)\right) .
\end{aligned}
$$

This completes the proof of 26 .

5. Completion of the estimate of $T_{2}$. Our first step is to replace the function $A_{f_{2}}(n)=\sum_{p \leq n} f_{2}(p) / p$ in the definition of $T_{2}$ by

$$
E_{f_{2}}(n)=\sum_{p^{\alpha} \leq n} \frac{f_{2}\left(p^{\alpha}\right)}{p^{\alpha}}\left(1-\frac{1}{p}\right)
$$

We have

$$
E_{f_{2}}(n)-A_{f_{2}}(n)=\sum_{\substack{p^{\alpha} \leq n \\ \alpha \geq 2}} \frac{f_{2}\left(p^{\alpha}\right)}{p^{\alpha}}-\sum_{p^{\alpha} \leq n} \frac{f_{2}\left(p^{\alpha}\right)}{p^{\alpha+1}}
$$

so that

$$
\left|E_{f_{2}}(n)-A_{f_{2}}(n)\right| \leq 2 K_{f_{2}}(n) \sum_{\substack{p^{\alpha} \leq n \\ \alpha \geq 2}} \frac{1}{p^{\alpha}}
$$

Observing that

$$
\sum_{\substack{p^{\alpha} \leq n \\ \alpha \geq 2}} \frac{1}{p^{\alpha}} \leq \sum_{p} \sum_{\alpha \geq 2} \frac{1}{p^{\alpha}}=\sum_{p} \frac{1}{p(p-1)} \leq \sum_{n \geq 2} \frac{1}{n(n-1)}=1,
$$

we obtain

$$
\left|E_{f_{2}}(n)-A_{f_{2}}(n)\right| \leq 2 K_{f_{2}}(n) .
$$

Using the inequality $|u+v|^{2} \leq 2|u|^{2}+2|v|^{2}$ with $u=f_{2}(a+b)-E_{f_{2}}(2 n)$ and $v=E_{f_{2}}(2 n)-A_{f_{2}}(2 n)$ (so that $|v| \leq 2 K_{f_{2}}(2 n)$ ), we get

$$
\begin{aligned}
\sum_{a \in \mathcal{A}} \sum_{b \in \mathcal{B}} \mid f_{2}(a & +b)-\left.A_{f_{2}}(2 n)\right|^{2} \\
& \leq 2 \sum_{a \in \mathcal{A}} \sum_{b \in \mathcal{B}}\left|f_{2}(a+b)-E_{f_{2}}(2 n)\right|^{2}+8|\mathcal{A}||\mathcal{B}| K_{f_{2}}^{2}(2 n)
\end{aligned}
$$


Now we will prove

$$
\sum_{a \in \mathcal{A}} \sum_{b \in \mathcal{B}}\left|f_{2}(a+b)-E_{f_{2}}(2 n)\right|^{2}=O\left(K_{f_{2}}^{2}(2 n)|\mathcal{A}||\mathcal{B}| \log \log (2 n)\right) .
$$

We have

$$
\begin{aligned}
\sum_{a \in \mathcal{A}} \sum_{b \in \mathcal{B}} \mid f_{2}(a+b) & -\left.E_{f_{2}}(2 n)\right|^{2} \\
= & S_{2}-S_{1} \overline{E_{f_{2}}(2 n)}-\overline{S_{1}} E_{f_{2}}(2 n)+|\mathcal{A}||\mathcal{B}|\left|E_{f_{2}}(2 n)\right|^{2},
\end{aligned}
$$

where $S_{1}$ and $S_{2}$ are defined by 21 and 27 respectively. We can rewrite this as

$$
\begin{aligned}
& \sum_{a \in \mathcal{A}} \sum_{b \in \mathcal{B}}\left|f_{2}(a+b)-E_{f_{2}}(2 n)\right|^{2} \\
&=\left(S_{2}-|\mathcal{A}||\mathcal{B}|\left|E_{f_{2}}(2 n)\right|^{2}\right)-\left(S_{1}-|\mathcal{A}||\mathcal{B}| E_{f_{2}}(2 n)\right) \overline{E_{f_{2}}(2 n)} \\
&-\left(\overline{S_{1}}-|\mathcal{A}||\mathcal{B}| \overline{E_{f_{2}}(2 n)}\right) E_{f_{2}}(2 n) .
\end{aligned}
$$

By Lemma 2 we have

$$
\left|S_{1}-\right| \mathcal{A}|| \mathcal{B}\left|E_{f_{2}}(2 n)\right| \ll K_{f_{2}}(2 n)|\mathcal{A}||\mathcal{B}|,
$$

and Lemma 3 yields

$$
\left.\left|S_{2}-\right| \mathcal{A}|| \mathcal{B}|| E_{f_{2}}(2 n)\right|^{2}\left|\ll K_{f_{2}}^{2}(2 n)\right| \mathcal{A}|| \mathcal{B} \mid \log \log (2 n),
$$

thus we obtain

$$
\begin{aligned}
\sum_{a \in \mathcal{A}} \sum_{b \in \mathcal{B}} \mid f_{2}(a+b) & -\left.E_{f_{2}}(2 n)\right|^{2} \\
& \ll K_{f_{2}}(2 n)\left(K_{f_{2}}(2 n) \log \log (2 n)+\left|E_{f_{2}}(2 n)\right|\right)|\mathcal{A}||\mathcal{B}| .
\end{aligned}
$$

Now observing that

$$
\begin{aligned}
\left|E_{f_{2}}(2 n)\right| & \leq\left|A_{f_{2}}(2 n)\right|+2 K_{f_{2}}(2 n) \\
& \leq K_{f_{2}}(2 n) \sum_{p \leq 2 n} \frac{1}{p}+2 K_{f_{2}}(2 n) \ll K_{f_{2}}(2 n) \log \log (2 n),
\end{aligned}
$$

we get 33 . It follows from 32 and 33 that

$$
T_{2}=O\left(K_{f_{2}}(2 n) \log \log (2 n)\right) .
$$

6. The estimate of $T_{1}$. Let $\omega_{V}(m)$ be the number of distinct prime factors of $m$ not exceeding $V$ :

$$
\omega_{V}(m)=\sum_{\substack{p \leq V \\ p \mid m}} 1
$$


Then for all $m \leq 2 n$ we have

$$
\left|f_{1}(m)\right|=\left|\sum_{\substack{p^{\alpha} \| m \\ p^{\alpha} \in \mathcal{P}_{1}}} f_{1}\left(p^{\alpha}\right)\right| \leq \sum_{\substack{p^{\alpha} \| m \\ p^{\alpha} \in \mathcal{P}_{1}}}\left|f_{1}\left(p^{\alpha}\right)\right|
$$

so that

$$
\left|f_{1}(m)\right| \leq K_{f_{1}}(2 n) \sum_{\substack{p^{\alpha} \| m \\ p^{\alpha} \in \mathcal{P}_{1}}} 1=K_{f_{1}}(2 n) \sum_{\substack{p \mid m \\ p \leq V}} 1=K_{f_{1}}(2 n) \omega_{V}(m) .
$$

Moreover by (8), (11) and (9) we have

$$
\begin{aligned}
\left|\sum_{p \leq 2 n} \frac{f_{1}(p)}{p}\right| & \leq K_{f_{1}}(2 n) \sum_{p \leq V} \frac{1}{p} \\
& \ll K_{f_{1}}(2 n) \log \log V \ll K_{f_{1}}(2 n) \log \log \log n .
\end{aligned}
$$

Using the inequality $\left|z_{1}+z_{2}\right|^{2} \leq 2\left(\left|z_{1}\right|^{2}+\left|z_{2}\right|^{2}\right)$ it follows that for $a \in \mathcal{A}$ and $b \in \mathcal{B}$,

$$
\begin{aligned}
\left|f_{1}(a+b)-\sum_{p \leq 2 n} \frac{f_{1}(p)}{p}\right|^{2} & \leq 2\left|f_{1}(a+b)\right|^{2}+2\left|\sum_{p \leq 2 n} \frac{f_{1}(p)}{p}\right|^{2} \\
& \leq 2 K_{f_{1}}^{2}(2 n) \omega_{V}^{2}(a+b)+O\left(K_{f_{1}}^{2}(2 n)(\log \log \log n)^{2}\right),
\end{aligned}
$$

so that

$$
\begin{aligned}
T_{1} & =\frac{1}{|\mathcal{A}||\mathcal{B}|} \sum_{a \in \mathcal{A}} \sum_{b \in \mathcal{B}}\left|f_{1}(a+b)-\sum_{p \leq 2 n} \frac{f_{1}(p)}{p}\right|^{2} \\
& \leq \frac{2 K_{f_{1}}^{2}(2 n)}{|\mathcal{A}||\mathcal{B}|} \sum_{a \in \mathcal{A}} \sum_{b \in \mathcal{B}} \omega_{V}^{2}(a+b)+O\left(K_{f_{1}}^{2}(2 n)(\log \log \log n)^{2}\right) .
\end{aligned}
$$

We split this double sum into two parts:

$$
T_{1} \leq 2 K_{f_{1}}^{2}(2 n)\left(X_{1}+X_{2}\right)
$$

where

$$
\begin{aligned}
& X_{1}=\frac{1}{|\mathcal{A}||\mathcal{B}|} \sum_{\substack{a \in \mathcal{A} \\
\omega_{V}(a+b) \leq 5 C(\log \log (2 n))^{1 / 2}}} \omega_{V}^{2}(a+b), \\
& X_{2}=\frac{1}{|\mathcal{A}||\mathcal{B}|} \sum_{\substack{a \in \mathcal{A} \\
\omega_{V}(a+b)>5 C(\log \log (2 n))^{1 / 2}}} \omega_{V}^{2}(a+b) . \\
& \sum_{b \in \mathcal{B}}
\end{aligned}
$$

Then clearly we have

$$
X_{1} \leq \frac{1}{|\mathcal{A}||\mathcal{B}|} \sum_{a \in \mathcal{A}} \sum_{b \in \mathcal{B}}\left(5 C(\log \log (2 n))^{1 / 2}\right)^{2}=25 C^{2} \log \log (2 n) .
$$


In order to estimate $X_{2}$, we may assume that (37)

$$
|\mathcal{A}| \leq|\mathcal{B}| .
$$

Then we have

$$
\begin{aligned}
X_{2} & =\frac{1}{|\mathcal{A}||\mathcal{B}|} \sum_{a \in \mathcal{A}} \sum_{\substack{b \in \mathcal{B} \\
\omega_{V}(a+b)>5 C(\log \log (2 n))^{1 / 2}}} \omega_{V}^{2}(a+b) \\
& \leq \frac{1}{|\mathcal{A}||\mathcal{B}|} \sum_{a \in \mathcal{A}} \sum_{\substack{m \leq 2 n \\
\omega_{V}(m)>5 C(\log \log (2 n))^{1 / 2}}} \omega_{V}^{2}(m) \\
& \leq \frac{1}{|\mathcal{B}|} \sum_{\substack{m \leq 2 n \\
\omega_{V}(m)>5 C(\log \log (2 n))^{1 / 2}}} \omega_{V}^{2}(m) .
\end{aligned}
$$

The last sum can be rewritten as

$$
\begin{aligned}
\sum_{\substack{m \leq 2 n \\
\omega_{V}(m)>5 C(\log \log (2 n))^{1 / 2}}} \omega_{V}^{2}(m) & =\sum_{t>5 C(\log \log (2 n))^{1 / 2}} \sum_{\substack{m \leq 2 n \\
\omega_{V}(m)=t}} t^{2} \\
& =\sum_{t>5 C(\log \log (2 n))^{1 / 2}} t^{2} \sum_{\substack{m \leq 2 n \\
\omega_{V}(m)=t}} 1 .
\end{aligned}
$$

Denote the smallest prime factor of a positive integer $i$ by $p(i)$ (and $p(1)=1$ ). If an integer $m$ is counted in the inner sum, then there are prime powers $q_{1}^{\alpha_{1}}, \ldots, q_{t}^{\alpha_{t}} \leq 2 n$ and an integer $r$ such that $q_{1}<\cdots<q_{t} \leq V, p(r)>V$ and $q_{1}^{\alpha_{1}} \cdots q_{t}^{\alpha_{t}} r=m(\leq 2 n)$. Thus the last sum in (39) is

$$
\begin{aligned}
& \sum_{\substack{m \leq 2 n \\
\omega_{V}(m)=t}} 1 \leq \sum_{\substack{\alpha_{1}^{\alpha_{1}}, \ldots, q_{t}^{\alpha_{t}} \\
q_{1}<\cdots<q_{t} \leq V}} \sum_{\substack{r \leq 2 n / q_{1}^{\alpha_{1}} \ldots q_{t}^{\alpha_{t}} \\
p(r)>V}} 1 \\
& \leq \sum_{\substack{\alpha_{1}, \ldots, q_{t}^{\alpha_{t}} \\
q_{1}<\cdots<q_{t} \leq V}} \sum_{r \leq 2 n / q_{1}^{\alpha_{1}} \ldots q_{t}^{\alpha_{t}}} 1 \\
& \leq \sum_{\substack{\alpha_{1}, \ldots, q_{t}^{\alpha_{t}} \\
q_{1}<\cdots<q_{t} \leq V}} \frac{2 n}{q_{1}^{\alpha_{1}} \cdots q_{t}^{\alpha_{t}}} \\
& \leq 2 n\left(\sum_{q \leq V, q^{\alpha} \leq 2 n} \frac{1}{q^{\alpha}}\right)^{t} \cdot \frac{1}{t !} \leq 2 n \frac{((1+o(1)) \log \log V)^{t}}{t !} .
\end{aligned}
$$

Inserting this estimate in $(39)$ we get

$$
\sum_{\substack{m \leq 2 n \\ \omega_{V}(m)>5 C(\log \log (2 n))^{1 / 2}}} \omega_{V}^{2}(m) \ll n \sum_{t>5 C(\log \log (2 n))^{1 / 2}} \frac{((1+o(1)) \log \log V)^{t}}{(t-2) !} .
$$


Hence, by the definition of $V$ given by (11), (9) and Stirling's formula, for large $n$ we have

$$
\begin{aligned}
& \sum_{\substack{m \leq 2 n \\
\omega_{V}(m)>5 C(\log \log (2 n))^{1 / 2}}} \omega_{V}^{2}(m) \\
& \ll n \sum_{t>5 C(\log \log (2 n))^{1 / 2}}((1 / 2+o(1)) \log \log \log (2 n))^{t}(3 / t)^{t-2} \\
& \ll n \exp \left(-(1+o(1)) 5 C(\log \log (2 n))^{1 / 2} \log \left(5 C(\log \log (2 n))^{1 / 2}\right)\right) \\
& \ll n \exp \left(-(1+o(1)) \frac{5}{2} C(\log \log (2 n))^{1 / 2} \log \log \log (2 n)\right),
\end{aligned}
$$

so that

$$
\begin{gathered}
\sum_{\substack{m \leq 2 n \\
\omega_{V}(m)>5 C(\log \log (2 n))^{1 / 2}}} \omega_{V}^{2}(m) \\
\ll n \exp \left(-2 C(\log \log (2 n))^{1 / 2} \log \log \log (2 n)\right) .
\end{gathered}
$$

It follows from (9), (37), (38) and (40) that

$$
\begin{aligned}
X_{2} & \ll \frac{n}{\sqrt{|\mathcal{A}||\mathcal{B}|}} \exp \left(-2 C(\log \log (2 n))^{1 / 2} \log \log \log (2 n)\right) \\
& \ll \exp \left(-C(\log \log (2 n))^{1 / 2} \log \log \log (2 n)\right)=o(1) .
\end{aligned}
$$

Combining (35), (36) and (41) we obtain

$$
T_{1}=O\left(C^{2} K_{f_{1}}^{2}(2 n) \log \log (2 n)\right) .
$$

7. The estimate of $T_{3}$ and the completion of the proof of Theorem 1. If $m \leq 2 n$ then

$$
\left|f_{3}(m)\right|=\left|\sum_{p^{\alpha} \| m} f_{3}\left(p^{\alpha}\right)\right| \leq \sum_{p^{\alpha} \| m}\left|f_{3}\left(p^{\alpha}\right)\right|,
$$

so that, since $f_{3}\left(p^{\alpha}\right)=0$ whenever $p^{\alpha} \notin \mathcal{P}_{3}$ and using (8),

$$
\left|f_{3}(m)\right| \leq \sum_{\substack{p^{\alpha} \in \mathcal{P}_{3} \\ p^{\alpha} \| m}} K_{f_{3}}(2 n)=K_{f_{3}}(2 n) \sum_{\substack{p^{\alpha} \in \mathcal{P}_{3} \\ p^{\alpha} \| m}} 1 .
$$

Here the last sum is $\leq 3$ since otherwise we would have

$$
m \geq \prod_{\substack{p^{\alpha} \in \mathcal{P}_{3} \\ p^{\alpha} \| m}} p^{\alpha}>\prod_{\substack{p^{\alpha} \in \mathcal{P}_{3} \\ p^{\alpha} \| m}} \sqrt[4]{2 n} \geq(\sqrt[4]{2 n})^{4}=2 n,
$$

which contradicts our assumption $m \leq 2 n$. Thus it follows from 43 that

$$
\left|f_{3}(a+b)\right| \leq 3 K_{f_{3}}(2 n) \quad \text { for all } a \in \mathcal{A}, b \in \mathcal{B} .
$$


Moreover

$$
\left|\sum_{p \leq 2 n} \frac{f_{3}(p)}{p}\right| \leq \sum_{p \leq 2 n} \frac{\left|f_{3}(p)\right|}{p} \leq K_{f_{3}}(2 n) \sum_{p \in \mathcal{P}_{3}} \frac{1}{p},
$$

so that

$$
\left|\sum_{p \leq 2 n} \frac{f_{3}(p)}{p}\right| \leq K_{f_{3}}(2 n) \sum_{\sqrt[4]{2 n}<p \leq 2 n} \frac{1}{p}=O\left(K_{f_{3}}(2 n)\right) .
$$

It follows from (44) and 45) that

$$
T_{3}=\frac{1}{|\mathcal{A}||\mathcal{B}|} \sum_{a \in \mathcal{A}} \sum_{b \in \mathcal{B}}\left|O\left(K_{f_{3}}(2 n)\right)+O\left(K_{f_{3}}(2 n)\right)\right|^{2}=O\left(K_{f_{3}}^{2}(2 n)\right) .
$$

(10) follows from (12), (34), 42 and (46), observing that $K_{f}(2 n)=$ $\max \left(K_{f_{1}}(2 n), K_{f_{2}}(2 n), K_{f_{3}}(2 n)\right)$, and this completes the proof of Theorem 1

Acknowledgements. The authors would like to thank the referee for the careful reading of the manuscript and his/her very helpful and valuable comments.

This research was partially supported by the Hungarian National Foundation for Scientific Research, Grants No K67676 and K72731, and by "Balaton" French-Hungarian exchange program F-48/06.

\section{References}

[1] A. Balog and A. Sárközy, On sums of sequences of integers. I, Acta Arith. 44 (1984), $73-86$.

[2] - - - On sums of sequences of integers. II, Acta Math. Hungar. 44 (1984), 169-179.

[3] - - - On sums of sequences of integers. III, ibid. 44 (1984), 339-349.

[4] W. D. Banks and I. E. Shparlinski, Prime divisors in Beatty sequences, J. Number Theory 123 (2007), 413-425.

[5] P. D. T. A. Elliott and A. Sárközy, The distribution of the number of prime divisors of sums $a+b$, ibid. 29 (1988), 94-99.

[6] P. Erdôs, H. Maier, and A. Sárközy, On the distribution of the number of prime factors of sums $a+b$, Trans. Amer. Math. Soc. 302 (1987), 269-280.

[7] P. Erdős, C. Pomerance, A. Sárközy, and C. L. Stewart, On elements of sumsets with many prime factors, J. Number Theory 44 (1993), 93-104.

[8] G. H. Hardy and S. Ramanujan, The normal number of prime factors of a number $n$, Quart. J. Math. 48 (1917), 76-92; also in: Collected Papers of Srinivasa Ramanujan, AMS Chelsea Publ., Providence, RI, 2000, 262-275.

[9] J. Kubilius, Probabilistic Methods in the Theory of Numbers, Transl. Math. Monogr. 11, Amer. Math. Soc., Providence, RI, 1964.

[10] H. L. Montgomery, The analytic principle of the large sieve, Bull. Amer. Math. Soc. 84 (1978), 547-567.

[11] A. Sárközy and C. L. Stewart, On divisors of sums of integers. II, J. Reine Angew. Math. 365 (1986), 171-191.

[12] - - -, On divisors of sums of integers. IV, Canad. J. Math. 40 (1988), 788-816. 
[13] P. Turán, On a theorem of Hardy and Ramanujan, J. London Math. Soc. 9 (1934), 274-276.

Joël Rivat

Institut de Mathématiques de Luminy

CNRS-UMR 6206

Université de la Méditerranée

163 avenue de Luminy, Case 907

13288 Marseille Cedex 9, France

E-mail: rivat@iml.univ-mrs.fr
András Sárközy Department of Algebra and Number Theory Eötvös Loránd University Pázmány Péter sétány $1 / \mathrm{c}$ H-1117 Budapest, Hungary E-mail: sarkozy@cs.elte.hu

Received on 7.11.2008

and in revised form on 12.10.2009 